\title{
Large area dynamic detection method for remote communication fault of power consumption information acquisition terminal
}

\author{
Yiqun Zhu' ${ }^{1}$, Jijun Zheng ${ }^{2 *}$, Zhen $\mathrm{Li}^{2}$ and Xiaolei $\mathrm{Liu}^{2}$ \\ ${ }^{1}$ Tianjin Marketing Service Center of State Grid (Measurement Center), Tianjin 300202, China \\ ${ }^{2}$ Yuanqi Industrial Technology Co. LTD., Qingdao, Shandong 266041, China
}

\begin{abstract}
With the rapid development of power system technology, the requirements for the stability of power equipment operation are higher and higher. In order to ensure the stability of power equipment operation, this paper puts forward the dynamic detection method of long-distance communication fault of power information acquisition terminal. Through the collection of remote communication fault dynamic information, data preprocessing. And the fault area is accurately located to determine the fault location. Through the power information acquisition terminal to determine the abnormal value, the detection effect of communication fault can be realized. According to the experimental comparison, it can be seen that compared with the traditional fault detection methods, the large interval dynamic detection method of power information acquisition terminal remote communication fault has higher detection accuracy and significant advantages.
\end{abstract}

\section{Introduction}

With the development of communication technology, computer network technology and big data industry, human beings have entered the information age. Information and communication technology promotes the strengthening of the trend of global economic and trade integration, and also brings more convenience to people's work, study and life ${ }^{[1]}$. In turn, the internal needs of human economic and trade exchanges and information communication are increasingly dependent on the update, iteration, optimization and upgrading of communication technology. With the increase of communication signal density, the signal characteristics become extremely complex, the signal transmission duration is different, and the frequency band becomes wider $^{[2]}$. In the high-speed communication mode, it needs to complete the signal band search, positioning and analysis in a very short time, which requires higher efficiency of communication information and stronger real-time signal transmission ${ }^{[3]}$. Based on this, this paper studies the dynamic detection method of long-range communication fault of power consumption information acquisition terminal, so as to better promote the transformation of traditional single channel communication mode to multi-channel communication mode.

\section{Long range dynamic detection of telecommunication fault}

\subsection{Long distance communication fault large area dynamic information collection.}

The module is mainly composed of five modules, in which the user login module is responsible for the safe use of the whole system to avoid the invasion of illegal users, the data management module is responsible for the data transmission with the external network to realize the collection and management of network test data, and the model management module can be used to build various special tests through model design, diagnosis and maintenance for network fault detection ${ }^{[4]}$. The fault detection module is composed of fault detection and detection results, which can be used to analyze the running state of remote communication of mobile acquisition terminal, and give the specific optimization scheme. The management module is mainly responsible for the user management, to ensure that it can be used in a safe environment. In the process of remote communication fault detection, the amount of data is relatively large, and part of the data structure involved is relatively complex ${ }^{[5]}$. If the structure design of data in the database is not scientific, the final efficiency of data mining will be affected to a certain extent. Oracle103 is used for remote communication fault detection. The database is used to store the relevant knowledge of remote communication fault, fault samples, fault occurrence rules, equipment operation status, production

\footnotetext{
*E-mail: zhengjijun11@163.com
} 
data and detailed information of fault maintenance personnel $^{[6]}$. Fault sample library refers to the process of remote communication fault detection, which adopts data mining technology to mine effective rules from massive production data and real-time status data list. The signal preprocessor is responsible for preprocessing the signals collected by the collector. In the process of fault detection, a series of digital signals can be obtained after the signal is converted by high-speed ADC. The signal is input into buffer RAM after frame processing, and the high-efficiency processing is realized by TMS320C5402 processor. In the high-precision DAC converter, the processed signal is input, the analog signal is restored, and the power amplifier is used to amplify the output, which ensures that the preprocessing result is more accurate and can effectively dig out the hidden law of the fault ${ }^{[7]}$. Compared with the host log data, the type and quantity of network data are more complex and huge, which makes the remote communication fault detection of network attacks more difficult. In order to integrate and analyze network packets effectively, we must understand a series of TCP / IP structures. The model of TCP / P protocol is simplified, and the combination of protocol stack is given. TPC and PI packet format, respectively, carry the key messages needed for analysis and processing. The original data packet collected by the data acquisition module is decrypted through the Ethernet frame to determine whether the protocol type belongs to the same IP address. If there is, the PI data layer parsing needs to be handed over to the IP parsing packet; otherwise, it will be discarded immediately. This paper deals with the electronic circuit fault in communication, and the processing flow is shown in Figure 1.

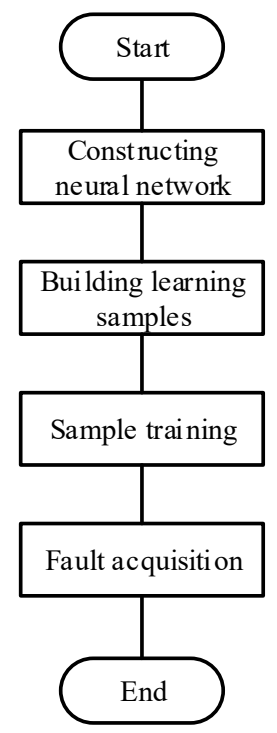

Fig. 1 Fault collection process

\subsection{Communication fault location algorithm}

In the design of the overall work flow of multi-channel communication signal fault detection, this paper adopts a master-slave handshake protocol mode and flow of control and terminal. The terminal workstation will upload the collected network node information to the information acquisition module. The acquisition module completes the data format conversion, noise reduction and other preprocessing, carries out broadcast communication, collects the fault information of multichannel communication network, and transmits the instructions of the upper computer chip down. Centralized analysis and processing of the collected fault information, and determine the location, area and type of communication network fault. With the master-slave handshake protocol flow of communication fault detection, on the one hand, the location of communication network fault can be verified, that is, whether the communication fault occurs on the node or on the $\operatorname{link}^{[8]}$. On the other hand, even if there are counting errors and network delay faults in the communication network, they can be completely and accurately identified. The segmentation feature extraction of irregular fault data can extend the processing time of the whole extraction operation by stabilizing the data redundancy dimension. On this basis, the sample space of the fault data to be extracted is continuously expanded, so as to fundamentally reduce the irregular fault data The purpose of the overall dimension. Let $\mathrm{f}$ represent a segmented set of $\mathrm{n}$ fault control data, then the irregularity suppression factor coefficient of each set can be expressed as:

$$
\left\{\begin{array}{l}
d_{0}=\hat{\chi}^{2} \frac{\gamma f_{0}}{n-0+\varepsilon} \\
d_{1}=\hat{\chi}^{2} \frac{\gamma f_{1}}{n-1+\varepsilon} \\
\vdots \\
d_{n}=\hat{\chi}^{2} \frac{\gamma f_{n}}{n-n+\varepsilon}
\end{array}\right.
$$

Where, $d$ represents $n$ different irregular inhibition factor coefficients, $\hat{\chi}$ representative coefficient acquisition parameters, $\gamma$ represents the weighted vector of factor coefficients, $\varepsilon$ is a fixed weighted period. The segmentation feature extraction results of fault data can be expressed as follows:

$$
k(n)=\sum_{l=1}^{d_{n}} \varphi(d) l(n-1)
$$

Where, $\varphi(d)$ represents the definition function related to the average number of $\mathrm{n}$ irregular suppression factor coefficients, and 1 represents the standard feature extraction operator. The network structure includes a database server and a network terminal server. The database server is mainly installed in the Oracle database, and the servers are all installed in Apache server and deployed on the corresponding network page for the business logic in the foreground page ${ }^{[9]}$. Axis 2 server is deployed in the network terminal server. Both database server and network terminal server are public servers. The required database server is public server, which is connected by router, and the servers connected by different routers are located in the subnet. According to the structure diagram, the communication fault is quickly 
located. According to the division stage and selection stage, the fault location workflow is designed, as shown in Figure 2.

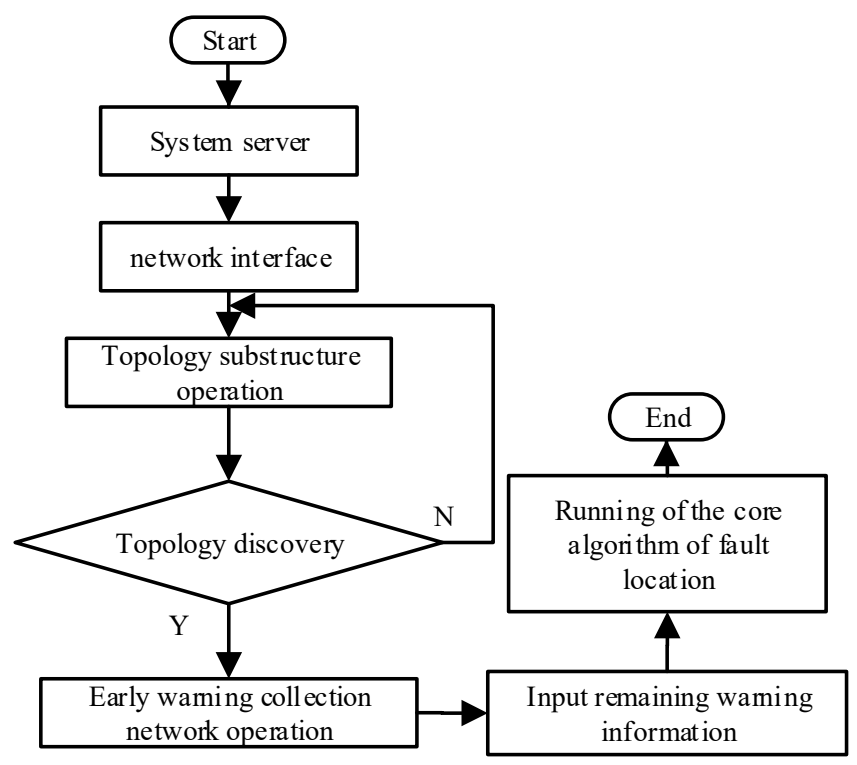

Fig. 2 Fault location workflow

The purpose of fault location algorithm is to find all possible faults and ensure the normal operation of remote communication of acquisition terminal ${ }^{[10]}$. According to the process shown in Figure 2, the specific analysis of its steps is as follows: obtain two warning information in any warning set; find the node that can explain the two warning information at the same time; use these nodes to replace the original warning information; repeat the acquisition Warning information, until there is no intersection description between the two warning information ${ }^{[11]}$. The signal model is designed by taking $\mathrm{x}$ and $\mathrm{y}$ as the signal acquisition module to collect the transmitted signal and the received signal.

$$
\left\{\begin{array}{l}
x(t)=s(t)+n(t) \\
y(t)=\sum_{k} \tau_{k} s(t-d)+n(t)
\end{array}\right.
$$

Where $s(t)$ is the original signal, $k$ is the sampling times, $\mathrm{E}$ is the coefficient of the original signal of the multi-channel communication network, and $n(t)$ is the function of the environmental noise of the fault detection.
Cross correlation operation is performed between the transmitted signal $x(t)$ and the received signal $y(t)$, then:

$$
\mathrm{R}_{\mathrm{xy}}(\mathrm{s})=\mathrm{E}[\mathrm{x}(\mathrm{t}) \mathrm{y}(\mathrm{t}+\mathrm{s})]_{(4)}
$$

Considering the environmental noise, the correlation between the two signals is rewritten as:

$$
\left\{\begin{array}{l}
R_{x y}(s)=\sum_{k} \tau_{k} R(s-d) \\
|R(s-d)| \leqslant R(0)
\end{array}\right.
$$

The correlation between the transmitted signal and the received signal in the multi-channel communication network is identified. Based on the quadratic correlation algorithm, the accuracy of network delay estimation can be improved under the condition of low signal-to-noise ratio, and the detailed information contained in the multi-channel communication fault signal can be accurately extracted. Therefore, the block diagram of dynamic fault detection is shown in Figure 3.

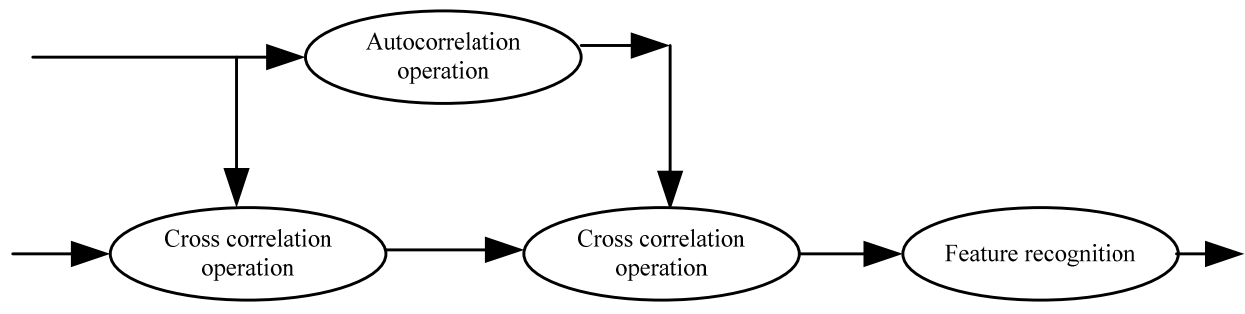

Fig. 3 block diagram structure of dynamic detection in fault area

Due to the frequency difference between multichannel communication signal and noise signal, it can be assumed that the useful signal is not correlated with noise signal in fault signal feature recognition:

$$
R_{x y}(\varsigma)=E\left[R_{x x}(t) R_{x y}(t+\mathrm{s})\right]=E\left[R_{\mathrm{s}}(t) ? \sum_{k} \tau_{k} R_{\mathrm{s}}(t-d)\right]=\sum \tau_{k} R_{k_{-}}(t-d)
$$

When the peak value $\mathrm{t}=\mathrm{d}$ appears in the correlation calculation, the delay of communication signal can be accurately estimated, and the detailed features of fault 
signal in multi-channel communication network can be accurately extracted, so as to realize the location and identification of fault node or link, and eliminate the communication fault of multi-channel network in time ${ }^{[12]}$. After the normal communication module sends the message to the optimal communication module, the other sub modules recursively forward the message to all other sub modules, and finally get most of the same messages as the sub modules. WiFi is used for communication between communication modules, and fault detection mechanism can prevent error information from entering, so as to avoid wrong operation and congestion of communication protoco ${ }^{[13]}$. In optical communication, fault detection algorithm needs to complete the following three tasks. The detection scheme must ensure that all communication subsystems (communication module, optical signal) complete the normal message processing. The normal sub can retrieve the correct signal according to the detection scheme. If a sub works normally, the rest of the communication network can receive the correct optical signal. In order to improve the accuracy and efficiency of pattern recognition, neural network algorithm is selected to detect the error information in the communication process. Using the labeled communication network training set elements as reference, the difference between the network information and the real port label information can be detected to generate the prediction area information. Then, back propagation algorithm is used to adjust the weight and offset of the network. After enough training, the neural network can successfully detect the error signal in the optical communication network.

\subsection{The realization of remote communication fault interval detection}

Remote communication fault detection based on data mining relies on the implicit assumption, that is, the task of switching from a failed server to another running server. Therefore, the remote communication transmission fault data mining is divided into $\mathrm{K}$ groups and e groups, each group contains at least one object. Therefore, based on the situation of $\mathrm{K}$ failure and $\mathrm{e}$ taking over its task, the data mining of transmission failure data in remote communication is divided into $\mathrm{K}$ group and E group, and this exchange mechanism is described in detail. In the case of no fault, the $\mathrm{K}$ and $\mathrm{e}$ servers receive the sensor and all the packets sent by the sensor ${ }^{[14]}$. Only $\mathrm{K}$ responds to these packets, computes the necessary control packets and sends them to the specified actuator node. When e detects the missing data packet (indicating that $\mathrm{K}$ is faulty), it enters a cycle to replace the inactive $\mathrm{K}$ and send the control data packet to the designated actuator. The control process running on $\mathrm{E}$ and used to back up $\mathrm{K}$ in case of fault must be designed to adapt to the situation of missing data packet. Similarly, control cannot easily lose control packets, which is to overcome the possibility of losing at most one packet when switching between $\mathrm{K}$ and $\mathrm{E}$. In this case, a simple solution is the "keep previous samples" technique until a new control packet is received. The possibility of telecommunication failure can be represented by three possible events: contention conflict, bandwidth unavailability and channel error. Let $\mathrm{P}$ denote the failure probability caused by the above three possible events, then:

$$
p_{f}=p_{c}+\left(1-p_{.}\right)\left(1-p_{c}\right)(1-q)^{T_{r}}+p_{0}+p_{c} p_{0}\left(1-p_{0}\right)\left(1-p_{c}\right)(1-q)^{\tau_{r}}-p_{c}\left(1-p_{0}\right)\left(1-p_{c}\right)(1-q)^{T_{r}}-p_{0}\left(1-p_{0}\right)\left(1-p_{c}\right)(1-q)^{T_{i}}-p_{c} p_{0}
$$

Where $\mathrm{P}_{\mathrm{e}}$ is the error probability caused by the channel, $\mathrm{P}_{2}$ is the collision probability caused by contention, $\mathrm{q}$ is the probability that the base station accepts the bandwidth request, and $\mathrm{T}$ is the response time or waiting time. By rearranging, we can get the following results:

$$
\begin{aligned}
& p_{f}=\left(p_{c}+p_{0}\right)\left[1-\left(1-p_{c}\right)(1-q)^{T}\left(1-p_{0}\right)\right]+n\left(1+p_{c} p_{e}\right)-p_{c} p_{e}\left[1-\left(1-p_{c}\right)(1-q)^{T_{r}}\left(1-p_{0}\right)\right] p_{\mathrm{u}} \\
& =\left(1-p_{c}\right)(1-q)^{T_{\mathrm{r}}}\left(1-p_{\mathrm{e}}\right) p_{\infty}
\end{aligned}
$$

Where $p_{\mathrm{u}}$ is the probability that the bandwidth is not available, $p_{c e}$ is the probability due to collision and channel error

$$
\begin{aligned}
& p_{\mathrm{f}}=p_{\mathrm{u}}+p_{\mathrm{ce}}\left(1-p_{\mathrm{u}}\right) \\
& =p_{\mathrm{ce}}-p_{\mathrm{u}}\left(1+p_{\mathrm{ce}}\right)
\end{aligned}
$$

In order to ensure that all the data have strong practical reference value, we can quickly select and process the fault data by analyzing the quantitative detection operator. When the detection quantitative operator is less than 1 , the total amount of fault data to be detected is less than the physical bearing range of the irregular control fault data model, and these data can also be used to calculate the smoothing index. The selection and processing flow of specific fault data to be detected is shown in Figure 4. 


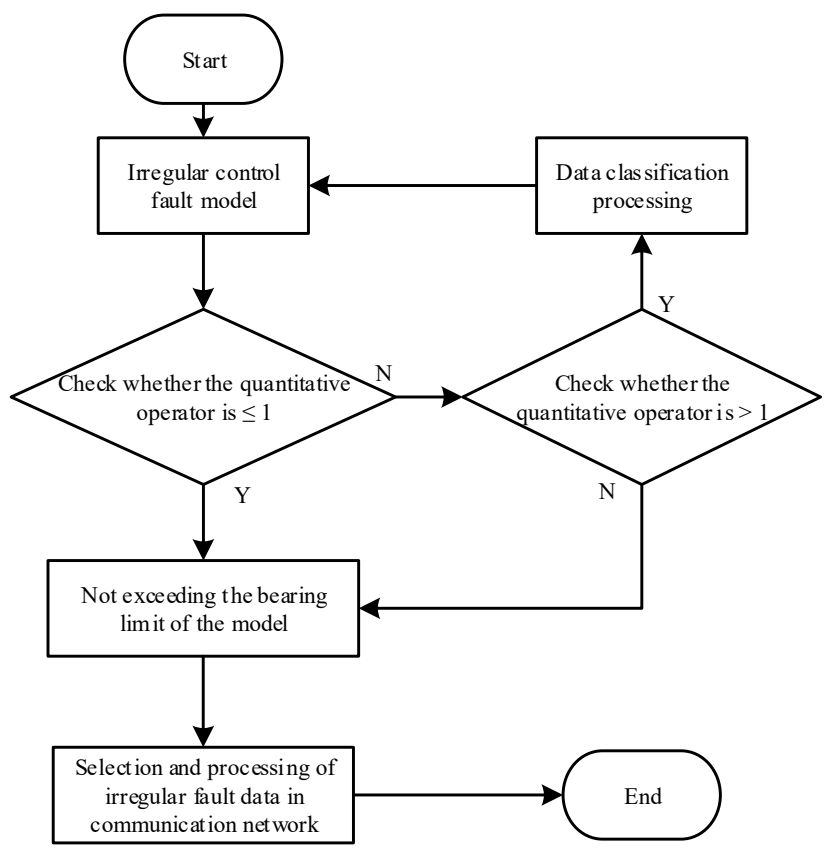

Fig. 4 Flow chart of fault data processing to be detected

In the process of fault detection, due to the characteristics of the equipment itself, the detection results contain a certain amount of noise. In order to improve the reliability of fault detection results, sub $\mathrm{GHz}$ technology should be used for the second noise reduction processing. Let $\mathrm{p}$ represent the basic physical period coefficient of real-time detection

$$
\mu=\frac{t^{\prime} y}{1-p}\left(S_{v}^{2}-S_{v^{\prime}}^{2}\right)
$$

Where y represents the standard detection vector of irregular control fault data, $S_{v} 、 S_{v^{\prime}}$ are represent the first-order detection operator and the second-order detection operator respectively, $v 、 v^{\prime}$ represent the operation coefficients of the irregular control fault data when the fixed-point operator is obtained. In order to reduce the noise figure as much as possible, the parasitic parameters generated by the parasitic capacitance in the structure are calculated. The $50 \mathrm{ohm}$ impedance is input to estimate the noise performance, and the maximum transmission power gain is achieved when the matching network is input, so that it can obtain better anti noise performance at lower working frequency. According to the noise reduction, the fault label can be obtained, and then the fault detection results of unknown samples can be obtained.

\section{Analysis of experimental results}

The maximum number of bytes allowed to be selected by the local host through TCP is 1024bytes 3.1.2. The local host protocol transmission control protocol (TCP) is the host environment through which frames are transmitted from the sender to the receiver. In order to ensure the accuracy of the experiment, the two remote communication fault detection methods are placed in the same test environment, using the same experimental data.
The experimental data information is shown in the table 1.

Tab. 1 Experimental data information

\begin{tabular}{ccc}
\hline Type & Number of data & Number of test data \\
\hline DoS & 500 & 100 \\
Probe & 200 & 100 \\
R2L & 100 & 40 \\
U2R0 & 50 & 10 \\
Normal & 1000 & 200 \\
\hline
\end{tabular}

The test process is carried out under the Windows XP platform, and the specific environment settings are shown in the table.

Tab. 2 Soft environment settings

\begin{tabular}{cc}
\hline Name & parameter \\
\hline Run host processor & Pentium.Dual-Core \\
& CPUE5400@2.70GHz \\
operation & Microsoft Windows XP \\
& Professional Version 2002 \\
& Service Pack3 \\
Computer acquisition files & Wav \\
Simulation experiment & 7.6.0.324(R2008a) \\
software platform & MATLAB
\end{tabular}

Using the network security packet tracking mode produced by a domestic company, the cycle is guaranteed to be 4 days, and the total data flow is $62 \mathrm{~GB}$. In order to facilitate the subsequent training and testing, this paper synthesizes the common network feature data stream and extracts the features. Table 3 and 4 shows the sample data for training and testing. 
Tab. 3 Test sample data

\begin{tabular}{cccc}
\hline Serial number & IP source address & Port number & state \\
\hline 1 & 119.25 .1 .15 & 1012 & normal \\
2 & 119.25 .1 .35 & 1251 & normal \\
3 & 119.25 .1 .103 & 3031 & normal \\
4 & 119.25 .2 .3 & 2008 & normal \\
\hline
\end{tabular}

Tab. 4 Test index

\begin{tabular}{ccc}
\hline $\begin{array}{c}\text { Fault } \\
\text { type }\end{array}$ & Monitoring items & $\begin{array}{c}\text { Early warning } \\
\text { conditions }\end{array}$ \\
\hline 1 & Cyclic redundancy code & For the first time \\
2 & TCP check error & $\begin{array}{c}\text { For the first time } \\
\text { Over threshold }\end{array}$ \\
3 & Fragment message & $\begin{array}{c}\text { First appearance, } \\
\text { exceeding threshold }\end{array}$ \\
4 & Retransmission message \\
\hline
\end{tabular}

20 mobile phones were selected for test and analysis, of which 10 were training samples and the other 10 were analysis samples. The sampling frequency was set at $40.2 \mathrm{kHz}$, and the sampling voltage range was [- 1,1] v. The specific parameters are set as follows: for each mobile phone, in the normal call mode, 10 segments of zero input, single frequency input and frequency mixing input are collected respectively, and $500 \mathrm{~ms}$ target data is selected from each segment; during frame processing, the frame length is set as $20 \mathrm{~ms}$, the acquisition frequency is $44.0 \mathrm{kHz}$, and there are about 526 data points in each frame; the data type is marked as- T. The others are labeled t. Based on the above steps, the fault detection effects of the traditional method and the method in this paper are compared and analyzed. The specific detection results are shown in the following figure.

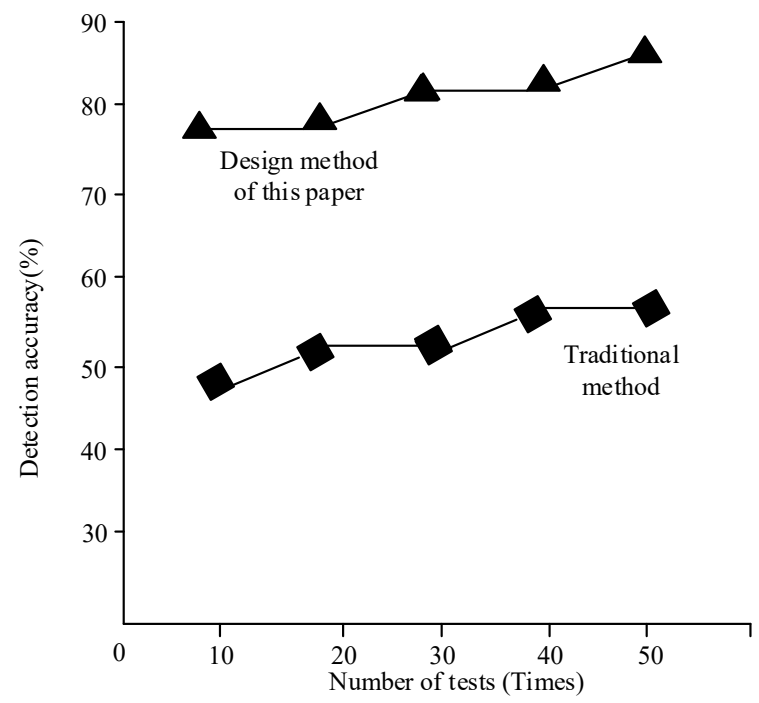

Fig. 5 Comparison test results

According to the comparison chart of the experimental results, it is obvious that compared with the traditional remote communication fault detection method, with the increase of the number of tests, the accuracy of the detection results of this method increases, and has been higher than the traditional method.

\section{Concluding}

In order to solve the problem of post fault analysis in communication fault detection, communication record and off-line analysis of digital power information terminal. This paper presents a method of large interval dynamic detection of power information acquisition remote communication fault. This method can automatically process a large number of remote communication information of the acquisition terminal, and deal with hidden dangers in time. In order to ensure the safe operation of power information terminal communication, the scientific basis of signal fault detection is provided. It provides a scientific basis for the development of power information terminal communication fault detection. Aiming at the optimization of power information terminal communication fault detection and testing, by analyzing the fault detection technology based on large interval dynamic detection of communication fault, the efficient detection of power information terminal communication fault can be realized under the simulation data test. This method can effectively shorten the network optimization time and reduce the maintenance cost for the operation.

\section{References}

1. Rodriguez-Frias M A , Yang W . Dual-Modality 4Terminal Electrical Capacitance and Resistance Tomography for Multiphase Flow Monitoring[J]. IEEE Sensors Journal, 2019, PP(99):1-1.

2. Ye H, Yang Y, Zhu L . A wireless network detection and control system for intelligent agricultural greenhouses based on NB-IOT technology[J]. Journal of Physics: Conference Series, 2021, 1738(1):012058 (9pp).

3. Zhang Y, Zhang Y, Zhao X, et al. Design and Data Analysis of Sports Information Acquisition System Based on Internet of Medical Things[J]. IEEE Access, 2020, PP(99):1-1.

4. Li Y, Jia H, Qi J , et al. An Acquisition Method of Agricultural Equipment Roll Angle Based on MultiSource Information Fusion[J]. Sensors, 2020, 20(7):2082.

5. Zhang B, Dou C, Yue D, et al. A Cyber-Physical Cooperative Hierarchical Control Strategy for Islanded Microgrid Facing With Random Communication Failure[J]. IEEE Systems Journal, 2020, PP(99):1-12.

6. Mithu, Sarkar, Bidyadhar, et al. Unified Smith Predictor Based $\mathrm{H}_{-} \infty$ Wide-Area Damping Controller to Improve the Control Resiliency to Communication Failure[J]. IEEE/CAA Journal of Automatica Sinica, 2020, v.7(02):273-285.

7. Masoud, H, Nazari, et al. Communication-FailureResilient Distributed Frequency Control in Smart Grids: Part I: Architecture and Distributed Algorithms[J]. IEEE Transactions on Power Systems, 2019, 35(2):1317-1326. 
8. Nazari M H , Wang L Y , Grijalva S, et al. Communication-Failure-Resilient Distributed Frequency Control in Smart Grids: Part II: Algorithmic Implementation and System Simulations[J]. IEEE Transactions on Power Systems, 2020, PP(99):1-1.

9. Aghaee, Dehkordi M, Bayati, et al. Distributed Control Methods and Impact of Communication Failure in AC Microgrids: A Comparative Review[J]. Electronics, 2019, 8(11):1265-1267.

10. $\mathrm{Yu}$ D, Chen C L P. Smooth Transition in Communication for Swarm Control With Formation Change[J]. IEEE Transactions on Industrial Informatics, 2020, 16(11):6962-6971.

11. Kim S, Kim C, Cho H , et al. A Hierarchical Routing Graph for Supporting Mobile Devices in Industrial Wireless Sensor Networks[J]. Sensors, 2021, 21(2):458.

12. Chen L, Choy Y S, Wang T G, et al. Fault detection of wheel in wheel/rail system using kurtosis beamforming method[J]. Structural health monitoring, 2020, 19(2):495-509.

13. Oliveira A K V D, Aghaei M, Ricardo Rüther. Aerial infrared thermography for low-cost and fast fault detection in utility-scale PV power plants[J]. Solar Energy, 2020, 211(4):712-724.

14. Sun S, Zhang H, Liu C, et al. Dissipativity-Based Intermittent Fault Detection and Tolerant Control for Multiple Delayed Uncertain Switched Fuzzy Stochastic Systems With Unmeasurable Premise Variables[J]. IEEE Transactions on Cybernetics, 2021, PP(99):1-15. 